\title{
Convergents of folded continued fractions
}

\author{
by \\ Jean-Paul Allouche (Orsay), \\ AnNA Lubiw (Waterloo, Ont.), \\ Michel Mendès France (Talence), \\ Alfred J. van der Poorten (North Ryde, N.S.W.) \\ and JefFrey Shallit (Waterloo, Ont.)
}

1. Introduction. Let $t(X)=\sum_{k>-n} a_{k} X^{-k}$ be a formal Laurent series in $\mathbb{Q}\left(\left(X^{-1}\right)\right)$. Then it is well known that $t(X)$ may be expanded uniquely as a continued fraction $\left[a_{0}, a_{1}, a_{2}, \ldots\right]$, where $a_{i} \in \mathbb{Q}[X]$ for $i \geq 0$ and $\operatorname{deg} a_{i}>0$ for $i>0$. (This fact was apparently first proved by E. Artin in his thesis $[2, \S 12]$; for alternative treatments, see $[11,3,14]$.) The $a_{i}$ are called the partial quotients. These expansions share many of the properties of ordinary continued fractions for real numbers; for example, if we set $p_{-2}=0, p_{-1}=1$, $q_{-2}=1, q_{-1}=0$, and $p_{n}=a_{n} p_{n-1}+q_{n-2}, q_{n}=a_{n} q_{n-1}+q_{n-2}$ for $n \geq 0$, then

$$
\left[a_{0}, a_{1}, \ldots, a_{n}\right]=\frac{p_{n}}{q_{n}} .
$$

We call $p_{n} / q_{n}$ the $n$th convergent to the continued fraction for $t(X)$. The following three identities will prove useful $[7,16]$ :

$$
\begin{gathered}
{\left[a_{n}, a_{n-1}, \ldots, a_{1}\right]=\frac{q_{n}}{q_{n-1}},} \\
p_{n} q_{n-1}-p_{n-1} q_{n}=(-1)^{n+1}, \\
\frac{p_{n}}{q_{n}}=a_{0}+\sum_{0 \leq i<n} \frac{(-1)^{i}}{q_{i} q_{i+1}} .
\end{gathered}
$$

1991 Mathematics Subject Classification: Primary 11A55; Secondary 11J70.

Key words and phrases: continued fraction, convergent, formal Laurent series, formal power series, paperfolding, automatic sequence.

Research of the fifth author supported in part by a grant from NSERC Canada. 
Let $\varepsilon_{0}=1$ and $\varepsilon_{i}= \pm 1$ for $i \geq 1$. Define

$$
\begin{aligned}
& g_{\varepsilon}(X)=\sum_{i \geq 0} \varepsilon_{i} X^{-2^{i}}, \\
& h_{\varepsilon}(X)=X \sum_{i \geq 0} \varepsilon_{i} X^{-2^{i}} .
\end{aligned}
$$

If the choice of signs is arbitrary, or is clear from the context, we will often omit the subscript and simply write $g(X)$ and $h(X)$. Previous papers have discussed the continued fractions for $g_{\varepsilon}(X)$ and $h_{\varepsilon}(X)$; see, for example, $[19,8,10,12,20,4,21,15]$. It is known that, no matter what the choice of signs is, the continued fraction for $g_{\varepsilon}(X)$ has partial quotients in the set $\{0, X-1, X+1, X, X+2, X-2\}$, and the continued fraction for $h_{\varepsilon}(X)$ has partial quotients in the set $\{1, X,-X\}$; furthermore, explicit formulas are known for these partial quotients in terms of the choice of signs made. These explicit formulas use a description arising from iterated paperfolding [5].

In this paper, we examine the convergents to the continued fractions for $g(X)$ and $h(X)$. In particular, we prove the surprising fact that the denominators of the convergents are polynomials in $\mathbb{Z}[X]$ with coefficients $0,1,-1$.

Here is a brief outline of this paper. In Section 2, we recall the descriptions of the continued fractions for $g(X)$ and $h(X)$ in terms of the folding and perturbed symmetry maps. In Section 3, we recall some basic facts about continuants and prove some technical lemmas. In Section 4, we prove some theorems about the constant terms of the convergents to $g(X)$ and $h(X)$. In Section 5, we give a recursion relation for computing the denominators of the convergents and characterize their support. In Section 6, we show how to describe the convergents for $h(X)$ in terms of those for $g(X)$. In Section 7, we obtain further results using some simple Diophantine approximation properties.

2. Paperfolding and continued fractions. Let $w$ and $x$ be finite sequences over an additive group $G$, and define the folding map $f_{x}(w)=$ $w \cdot x \cdot\left(-w^{\mathrm{R}}\right)$, where $\cdot$ represents concatenation, $-w$ represents the same sequence as $w$, but with all signs reversed, and $w^{\mathrm{R}}$ represents the sequence $w$ taken in reverse order.

Let $G$ be a group written additively, and let $x_{i} \in G$ for $i \geq 1$. Set

$$
\mathcal{F}\left(x_{1}, x_{2}, \ldots, x_{k}\right)=f_{x_{k}}\left(f_{x_{k-1}}\left(\ldots\left(f_{x_{2}}\left(f_{x_{1}}(\lambda)\right)\right) \ldots\right)\right),
$$

where $\lambda$ represents the empty sequence. Since $\mathcal{F}\left(x_{1}, \ldots, x_{k}\right)$ is a prefix of the sequence $\mathcal{F}\left(x_{1}, x_{2}, \ldots, x_{k}, x_{k+1}\right)$, the limiting sequence $\mathcal{F}\left(x_{1}, x_{2}, \ldots\right)$ is 
well-defined. For example, $\mathcal{F}(1,2,3, \ldots)$ denotes the infinite sequence

$$
(1,2,-1,3,1,-2,-1,4,1, \ldots) \text {. }
$$

We now have the following

TheOrEm 1. If $\left(a_{1}, a_{2}, \ldots\right)=\mathcal{F}\left(x_{1}, x_{2}, \ldots\right)$, then

(a) $a_{2 i+1}=(-1)^{i} x_{1}$ for $i \geq 0$,

(b) $\left(a_{2 i}\right)_{i \geq 1}=\mathcal{F}\left(x_{2}, x_{3}, x_{4}, \ldots\right)$,

(c) $a_{2^{k}+j}=-a_{2^{k}-j}$ for $k \geq 1,0 \leq j \leq 2^{k}-1$.

Proof. The easy proof by induction is left to the reader.

If $e_{i}= \pm 1$ for $i \geq 1$, we say that $\mathcal{F}\left(e_{1}, e_{2}, \ldots\right)$ is a paperfolding sequence $[13,5]$. In this case, the $e_{i}$ are sometimes referred to as the unfolding instructions.

In the special case $e_{i}=1$ for $i \geq 1$, we get the regular paperfolding sequence

$$
\begin{aligned}
\left(s_{i}\right)_{i \geq 1} & =\mathcal{F}(1,1,1, \ldots) \\
& =(1,1,-1,1,1,-1,-1,1,1,1,-1,-1,1,-1,-1, \ldots) .
\end{aligned}
$$

For this sequence, we have

$$
s_{n}=(-1)^{r}
$$

if $n=2^{a}(2 r+1)$.

Now let $h(X)=X \sum_{i \geq 0} \varepsilon_{i} X^{-2^{i}}$, and assume $\varepsilon_{0}=1$, and $\varepsilon_{i}= \pm 1$ for $i \geq 1$. It is known [15] that

$$
h(X)=\left[1, X \mathcal{F}\left(\varepsilon_{1},-\varepsilon_{2},-\varepsilon_{3},-\varepsilon_{4}, \ldots\right)\right] .
$$

For example, we have

$$
X \sum_{k \geq 0} X^{-2^{k}}=[1, X,-X,-X,-X, X, X,-X,-X, \ldots] .
$$

Similarly, we can define the perturbed symmetry map $p_{x}(w)=w \cdot x \cdot w^{\mathrm{R}}$ [12]. Let $x_{1}, \ldots, x_{k}$ each represent a finite sequence over an additive group $G$. Then we define

$$
\mathcal{P}\left(x_{1}, x_{2}, \ldots, x_{k}\right)=p_{x_{k}}\left(p_{x_{k-1}}\left(\ldots\left(p_{x_{2}}\left(p_{x_{1}}(\lambda)\right)\right) \ldots\right)\right) .
$$

As before, we can also consider the limiting sequence $\mathcal{P}\left(x_{1}, x_{2}, \ldots\right)$. It is known [4, Theorem 5] that if $g(X)=\sum_{i \geq 0} \varepsilon_{i} X^{-2^{i}}$, then

$$
g(X)=\left[0, X-\varepsilon_{1}, \mathcal{P}\left(c_{2}, c_{3}, c_{4}, \ldots\right)\right],
$$

where

$$
c_{2}=\left(X+\varepsilon_{1}+\varepsilon_{2}, X+\varepsilon_{1}-\varepsilon_{2}\right)
$$


and

$$
c_{j}=\left(X-\varepsilon_{1}+\varepsilon_{j}, X-\varepsilon_{1}-\varepsilon_{j}\right) \quad \text { for } j \geq 3 .
$$

For example, we have

$$
\sum_{k \geq 0} X^{-2^{k}}=[0, X-1, X+2, X, X, X-2, X, X+2, \ldots] .
$$

3. Continuants. We recall the basic definition of the multivariate polynomials known as continuants. Define $K_{0}()=1, K_{1}\left(a_{1}\right)=a_{1}$, and $K_{n}\left(a_{1}\right.$, $\left.\ldots, a_{n}\right)=a_{n} K_{n-1}\left(a_{1}, \ldots, a_{n-1}\right)+K_{n-2}\left(a_{1}, \ldots, a_{n-2}\right)$. It is also convenient to set $K_{-1}()=0$. Then it is not difficult to see that

$$
\left[a_{0}, a_{1}, \ldots, a_{n}\right]=\frac{K_{n+1}\left(a_{0}, a_{1}, \ldots, a_{n}\right)}{K_{n}\left(a_{1}, \ldots, a_{n}\right)}
$$

for all $n \geq 0[9, \S 4.5 .3]$.

We now give three technical lemmas on continuants which will be used later in the paper.

Lemma 2. Let $n \geq 1$ and $a_{1}, \ldots, a_{n-1}$ be real numbers. Then

$$
\begin{aligned}
K_{n+j}\left(a_{1}, a_{2}, \ldots, a_{n-1}, 0,-a_{n-1},-a_{n-2}, \ldots,-a_{n-j}\right) \\
=K_{n-j-2}\left(a_{1}, a_{2}, \ldots, a_{n-j-2}\right)
\end{aligned}
$$

for $0 \leq j \leq n-1$.

Proof. The easy induction on $j$ is left to the reader.

Lemma 3. Let $n \geq 1$, and let $a_{1}, \ldots, a_{n}$ be integers. Then

$$
\begin{aligned}
K_{n+j}\left(a_{1}, \ldots, a_{n-1}, a_{n},-a_{n-1},-a_{n-2}, \ldots,-a_{n-j}\right) & \\
\equiv K_{n+j}\left(a_{1}, \ldots, a_{n-1}, 0,-a_{n-1},-a_{n-2}, \ldots,-a_{n-j}\right) & \left(\bmod a_{n} K_{n-1}\left(a_{1}, \ldots, a_{n-1}\right)\right)
\end{aligned}
$$

for $0 \leq j \leq n-1$.

Proof. Let $q_{n-1}=K_{n-1}\left(a_{1}, a_{2}, \ldots, a_{n-1}\right)$ and $q_{n-2}=K_{n-2}\left(a_{1}, a_{2}, \ldots\right.$ $\left.\ldots, a_{n-2}\right)$. Then we have

$$
\begin{aligned}
K_{n}\left(a_{1}, a_{2}, \ldots, a_{n-1}, a_{n}\right) & =a_{n} q_{n-1}+q_{n-2}, \\
K_{n}\left(a_{1}, a_{2}, \ldots, a_{n-1}, 0\right) & =q_{n-2} .
\end{aligned}
$$

Now for each $j$ there exist integer constants $c_{1}, c_{2}$ such that

$$
\begin{aligned}
K_{n+j}\left(a_{1}, \ldots, a_{n-1},\right. & \left.a_{n},-a_{n-1},-a_{n-2}, \ldots,-a_{n-j}\right) \\
& =c_{1} K_{n-1}\left(a_{1}, \ldots, a_{n-1}\right)+c_{2} K_{n}\left(a_{1}, \ldots, a_{n-1}, a_{n}\right)
\end{aligned}
$$

and

$$
\begin{aligned}
K_{n+j}\left(a_{1}, \ldots, a_{n-1}, 0\right. & \left.,-a_{n-1},-a_{n-2}, \ldots,-a_{n-j}\right) \\
= & c_{1} K_{n-1}\left(a_{1}, \ldots, a_{n-1}\right)+c_{2} K_{n}\left(a_{1}, \ldots, a_{n-1}, 0\right) .
\end{aligned}
$$


Hence, subtracting these last two equations, we get

$$
\begin{aligned}
& K_{n+j}\left(a_{1}, \ldots, a_{n-1}, a_{n},-a_{n-1},-a_{n-2}, \ldots,-a_{n-j}\right) \\
& \quad-K_{n+j}\left(a_{1}, \ldots, a_{n-1}, 0,-a_{n-1},-a_{n-2}, \ldots,-a_{n-j}\right)=c_{2} a_{n} q_{n-1},
\end{aligned}
$$

which proves the desired result.

Lemma 4. Let $k_{i} \geq 0$ for $i \geq 1$, and let

$$
c=\left(c_{1}, c_{2}, \ldots\right)=\left(-1,0^{k_{1}}, 2,0^{k_{2}},-2,0^{k_{3}}, 2,0^{k_{4}},-2,0^{k_{5}}, 2, \ldots\right)
$$

be an infinite sequence of integers. (The exponents on the 0 's refer to the number of repetitions, and the 2's alternate in sign.) Then for all $n \geq 0$, $K_{n}\left(c_{1}, \ldots, c_{n}\right)= \pm 1$, and the same result holds for $-c=\left(-1,0^{k_{1}},-2,0^{k_{2}}, 2\right.$, $\left.0^{k_{3}},-2, \ldots\right)$.

Proof. Let $q_{n}=K_{n}\left(c_{1}, \ldots, c_{n}\right)$. Then $q_{n}$ is the denominator of the $n$th convergent of the continued fraction

$$
\left[0,-1,0^{k_{1}}, 2,0^{k_{2}},-2,0^{k_{3}}, 2,0^{k_{4}},-2,0^{k_{5}}, 2, \ldots\right] .
$$

By (2), it follows that

$$
\frac{q_{n}}{q_{n+1}}=\overbrace{\left[\ldots,-2,0^{k_{2}}, 2,0^{k_{1}},-1\right.}^{n \text { terms }}] .
$$

But $\left[2,0^{k_{1}},-1\right]=1$ for all $k_{1} \geq 0$. It follows that $q_{n} / q_{n+1}=\left[\ldots,-2,0^{k_{2}}, 1\right]$. But $\left[-2,0^{k_{2}}, 1\right]=-1$ for all $k_{2} \geq 0$. It now follows by induction that $q_{n} / q_{n+1}= \pm 1$. Since $q_{0}=1$, we conclude that $q_{n}= \pm 1$ for all $n \geq 0$.

4. The constant term. Let $g_{\varepsilon}(X)$ and $h_{\varepsilon}(X)$ be as defined above in (5) and (6). Let $p_{n}^{\prime} / q_{n}^{\prime}$ be the $n$th convergent to the continued fraction for $g_{\varepsilon}(X)$, and $p_{n} / q_{n}$ be the $n$th convergent to the continued fraction for $h_{\varepsilon}(X)$. Then $\left(p_{n}^{\prime}\right)_{n \geq 0},\left(q_{n}^{\prime}\right)_{n \geq 0},\left(p_{n}\right)_{n \geq 0}$, and $\left(q_{n}\right)_{n \geq 0}$ are sequences of polynomials in $X$, and we are interested in their constant term.

We begin with the constant terms of $p_{n}$ and $q_{n}$ :

Theorem 5. For $n \geq-1$ we have

(a) $p_{n}(0)=1$

(b) $q_{2 n}(0)=1$;

(c) $q_{2 n+1}(0)=0$.

Pr o o f. Easy proof by induction on $n$, using the fact that $p_{n}= \pm X p_{n-1}+$ $p_{n-2}$, and $q_{n}= \pm X q_{n-1}+q_{n-2}$.

We now turn to the constant terms of $p_{n}^{\prime}$ and $q_{n}^{\prime}$.

Theorem 6. For $n \geq 0$ we have $q_{n}^{\prime}(0)= \pm 1$. 
Proof. Note that if $g_{\varepsilon}(X)=\left[a_{0}^{\prime}(X), a_{1}^{\prime}(X), \ldots\right]$, then by (10), we have $a_{i}^{\prime}(0)=c_{i}$, where $\left(c_{i}\right)$ is the sequence in Lemma 4 .

R e m a r k. Evidently the proof applies to any formal Laurent series such that the constant terms of the partial quotients of its continued fraction expansion is given by the sequence $\left(c_{i}\right)$ of Lemma 4 .

We now discuss $p_{n}^{\prime}$ and $q_{n}^{\prime}$ for a particular choice of $\varepsilon$; namely, when $\varepsilon_{i}=1$ for all $i \geq 0$. First, we describe the partial quotients for the continued fraction expansion of $\sum_{i \geq 0} X^{-2^{i}}$ :

Theorem 7. Let $\sum_{i>0} X^{-2^{i}}=\left[a_{0}^{\prime}(X), a_{1}^{\prime}(X), \ldots\right]$. Then $a_{1}^{\prime}(X)=X-1$, and $a_{n}^{\prime}(X)=X-(-1)^{\lfloor n / 2\rfloor}+(-1)^{n} s_{\lfloor n / 2\rfloor}$, where $\left(s_{n}\right)_{n \geq 1}$ is the regular paperfolding sequence of $(7)$.

Proof. Follows from (10).

Now we discuss the denominators of the convergents to $\sum_{i \geq 0} X^{-2^{i}}$ :

TheOREM 8. Let $q_{n}^{\prime}(X)$ be the denominator of the nth convergent to $\sum_{i \geq 0} X^{-2^{i}}$. Then

$$
q_{n}^{\prime}(0)=(-1)^{n+\lfloor n / 2\rfloor} s_{1} \ldots s_{n},
$$

where $\left(s_{i}\right)_{i \geq 1}$ is the regular paperfolding sequence of $(7)$.

Proof. By induction, using the fact that $q_{n+1}^{\prime}(0)=a_{n+1}^{\prime}(0) q_{n}^{\prime}(0)+$ $q_{n-1}^{\prime}(0)$. (Recall that the sequence $a_{n+1}^{\prime}$ was described in Theorem 7.)

Now we discuss the numerators of the convergents to $\sum_{i \geq 0} X^{-2^{i}}$ :

Theorem 9. For $n \geq 0$ we have

$$
\frac{p_{n}^{\prime}(0)}{q_{n}^{\prime}(0)}=-\sum_{1 \leq i \leq n} s_{i}
$$

where $\left(s_{i}\right)_{i \geq 1}$ is the regular paperfolding sequence of (7).

Proof. By (4) we know that

$$
\frac{p_{n}^{\prime}(0)}{q_{n}^{\prime}(0)}=\frac{1}{q_{0}^{\prime}(0) q_{1}^{\prime}(0)}-\frac{1}{q_{1}^{\prime}(0) q_{2}^{\prime}(0)}+\ldots+\frac{(-1)^{n-1}}{q_{n-1}^{\prime}(0) q_{n}^{\prime}(0)} .
$$

Since $q_{n}^{\prime}(0)= \pm 1$ by Theorem 6 , it follows that $q_{n}^{\prime}(0)=1 / q_{n}^{\prime}(0)$, and we get

$$
\begin{aligned}
\frac{p_{n}^{\prime}(0)}{q_{n}^{\prime}(0)}= & q_{0}^{\prime}(0) q_{1}^{\prime}(0)-q_{1}^{\prime}(0) q_{2}^{\prime}(0) \\
& +q_{2}^{\prime}(0) q_{3}^{\prime}(0)-\ldots+(-1)^{n-1} q_{n-1}^{\prime}(0) q_{n}^{\prime}(0) .
\end{aligned}
$$


Let us first consider the case where $n$ is even, say $n=2 k$. Then (12) and Theorem 8 gives

$$
\begin{aligned}
\frac{p_{2 k}^{\prime}(0)}{q_{2 k}^{\prime}(0)} & =-a_{2}^{\prime}(0)-a_{4}^{\prime}(0)-\ldots-a_{2 k}^{\prime}(0) \\
& =-\sum_{1 \leq i \leq k} a_{2 i}^{\prime}(0)=\sum_{1 \leq i \leq k}(-1)^{i}-\sum_{1 \leq i \leq k} s_{i} \\
& =-\sum_{0 \leq i \leq k-1} s_{2 i+1}-\sum_{1 \leq i \leq k} s_{2 i}=-\sum_{1 \leq i \leq 2 k} s_{i} .
\end{aligned}
$$

Now let us consider the case where $n$ is odd, say $n=2 k+1$. Then by (12) and Theorem 8 we get

$$
\begin{aligned}
\frac{p_{2 k+1}^{\prime}(0)}{q_{2 k+1}^{\prime}(0)} & =\frac{p_{2 k}^{\prime}(0)}{q_{2 k}^{\prime}(0)}+q_{2 k}^{\prime}(0) q_{2 k+1}^{\prime}(0) \\
& =\frac{p_{2 k}^{\prime}(0)}{q_{2 k}^{\prime}(0)}+\left((-1)^{k} s_{1} s_{2} \ldots s_{2 k}\right)\left((-1)^{k+1} s_{1} s_{2} \ldots s_{2 k+1}\right) \\
& =\frac{p_{2 k}^{\prime}(0)}{q_{2 k}^{\prime}(0)}-s_{2 k+1}=-\sum_{1 \leq i \leq 2 k+1} s_{i} .
\end{aligned}
$$

The result now follows.

Remark. It is easy to prove by induction that $\sum_{1 \leq k \leq n} s_{k}$ is always positive, and so we have $\operatorname{sgn} p_{n}^{\prime}(0)=-q_{n}^{\prime}(0)$.

Rem ark. In true Apéry fashion, we have therefore established that the best approximations to $\sum_{k \geq 0} 1 / 0^{2^{k}}$ are the integers $-\left|p_{n}^{\prime}(0)\right|$.

Our result also implies that the sequence $p_{n}^{\prime}(0)$ is 2-regular in the sense of Allouche and Shallit [1].

EXERCiSE. Prove that $\left|p_{n}^{\prime}(0)\right|=O(\log n)$. Also show that, for $k \geq 0$, the base-2 representation of the least index $n$ such that $\left|p_{n}^{\prime}(0)\right|=k$ is $\overbrace{10101 \ldots}^{k \text { symbols }}$

ExERCiSE. Show that the coefficient of the second-highest term in $q_{n}^{\prime}(X)$ is $(-1)^{n} s_{n}$ for $n \geq 1$.

5. More on convergents. In the previous section, we studied the constant term of the convergents to $g_{\varepsilon}(X)=\sum_{i \geq 0} \varepsilon_{i} X^{-2^{i}}$. Our results suggest studying the rest of the coefficients of the convergents. To aid intuition, in Table 1 we list the convergents $p_{n}^{\prime}(X) / q_{n}^{\prime}(X)$ to $\sum_{i \geq 0} X^{-2^{i}}$.

The observant reader will note that the coefficients of $q_{n}^{\prime}(X)$ lie in $\{0,1,-1\}$ for $0 \leq n \leq 9$ and will naturally wonder if this is true for all $n$. We prove this (and more) below in Corollary 16. For the moment, however, 
Table 1. Convergents to $\sum_{i \geq 0} X^{-2^{i}}$

\begin{tabular}{|l|l|l|l|}
\hline$n$ & $a_{n}^{\prime}(X)$ & $p_{n}^{\prime}(X)$ & $q_{n}^{\prime}(X)$ \\
\hline 0 & 0 & 0 & 1 \\
1 & $X-1$ & 1 & $X-1$ \\
2 & $X+2$ & $X+2$ & $X^{2}+X-1$ \\
3 & $X$ & $X^{2}+2 X+1$ & $X^{3}+X^{2}-1$ \\
4 & $X$ & $X^{3}+2 X^{2}+2 X+2$ & $X^{4}+X^{3}+X^{2}-1$ \\
5 & $X-2$ & $X^{4}-X^{2}-3$ & $X^{5}-X^{4}-X^{2}-X+1$ \\
6 & $X$ & $X^{5}+2 X^{2}-X+2$ & $X^{6}-X^{5}+X^{4}+X-1$ \\
7 & $X+2$ & $X^{6}+2 X^{5}+X^{4}+2 X^{3}+2 X^{2}+1$ & $X^{7}+X^{6}+X^{4}-1$ \\
8 & $X$ & $X^{7}+2 X^{6}+2 X^{5}$ & $X^{8}+X^{7}+X^{6}+X^{4}-1$ \\
& & & $+2 X^{4}+2 X^{3}+2 X^{2}+2$ \\
9 & $X-2$ & $X^{8}-X^{6}-X^{4}-2 X^{2}+2 X-3$ & $X^{9}-X^{8}-X^{6}+X^{5}-X^{4}-X+1$ \\
\hline
\end{tabular}

we turn to the study of $h_{\varepsilon}(X)=X g_{\varepsilon}(X)$ instead. This series is somewhat easier to handle and, as we will see in Section 6 , the results we obtain for $h_{\varepsilon}(X)$ will also imply results for $g_{\varepsilon}(X)$.

Again, to build intuition, we provide a brief table of the convergents $p_{n}(X) / q_{n}(X)$ to $X \sum_{i \geq 0} X^{-2^{i}}$.

Table 2. Convergents to $X \sum_{i \geq 0} X^{-2^{i}}$

\begin{tabular}{|r|r|l|l|}
\hline$n$ & $a_{n}(X)$ & $p_{n}(X)$ & $q_{n}(X)$ \\
\hline 0 & 1 & 1 & 1 \\
1 & $X$ & $X+1$ & $X$ \\
2 & $-X$ & $-X^{2}-X+1$ & $-X^{2}+1$ \\
3 & $-X$ & $X^{3}+X^{2}+1$ & $X^{3}$ \\
4 & $-X$ & $-X^{4}-X^{3}-X^{2}-2 X+1$ & $-X^{4}-X^{2}+1$ \\
5 & $X$ & $-X^{5}-X^{4}-X^{2}+X+1$ & $-X^{5}+X$ \\
6 & $X$ & $-X^{6}-X^{5}-X^{4}-2 X^{3}-X+1$ & $-X^{6}-X^{4}+1$ \\
7 & $-X$ & $X^{7}+X^{6}+X^{4}+1$ & $X^{7}$ \\
8 & $-X$ & $-X^{8}-X^{7}-X^{6}-2 X^{5}-X^{4}-2 X^{3}-2 X+1$ & $-X^{8}-X^{6}-X^{4}+1$ \\
9 & $X$ & $-X^{9}-X^{8}-X^{6}-X^{5}-X^{4}-2 X^{2}+X+1$ & $-X^{9}-X^{5}+X$ \\
\hline
\end{tabular}

We begin our investigation by studying the denominators $q_{n}(X)$ of convergents to $h_{\varepsilon}(X)$. Unfortunately, the notation $q_{n}(X)$ is no longer sufficient, so we introduce the new notation $q_{n}^{\left(a_{1}, a_{2}, \ldots\right)}(X)$ to make the dependence on the partial quotients clear. More precisely, we define

$$
q_{n}^{\left(a_{1}, a_{2}, \ldots\right)}(X)=K_{n}\left(a_{1}, \ldots, a_{n}\right) .
$$

(Since $q_{n}$ does not depend on $a_{0}$, we do not specify it explicitly.)

Now let $e_{i}= \pm 1$ for $i \geq 1$, and define $e=\left(e_{1}, e_{2}, \ldots\right)$. By (9), we know that $q_{n}^{X \mathcal{F}(e)}(X)$ is the denominator of the $n$th convergent to $h_{\varepsilon}(X)$, where $\varepsilon_{0}=1, \varepsilon_{1}=e_{1}$, and $\varepsilon_{i}=-e_{i}$ for $i \geq 2$. We now give a recursive formula 
for $q_{n}^{X \mathcal{F}(e)}(X)$ that expresses it as the sum of two polynomials, one with high-order terms and one with low-order terms.

Theorem 10. Write $n=2^{k}+j$, with $k \geq 0$ and $0 \leq j \leq 2^{k}-1$. Let $e_{i}= \pm 1$ for $i \geq 1$. Define $e=\left(e_{1}, e_{2}, \ldots\right)$ and $e^{\prime}=\left(e_{1}, e_{2}, \ldots, e_{k-1},-e_{k}\right)$. Let $\mathcal{F}(e)=\left(a_{1}, a_{2}, \ldots\right)$. Then

$$
\begin{aligned}
& q_{n}^{X \mathcal{F}(e)}(X) \\
& \quad= \begin{cases}e_{1} X & \text { if } n=1, \\
-e_{k+1} X^{2^{k+1}}-1 & \text { if } k \geq 1 \text { and } j=2^{k}-1, \\
-e_{k} e_{k+1} X^{2^{k}} q_{j}^{X \mathcal{F}\left(e^{\prime}\right)}(X)+q_{2^{k}-j-2}^{X \mathcal{F}(e)}(X) & \text { if } k \geq 1 \text { and } 0 \leq j \leq 2^{k}-2 .\end{cases}
\end{aligned}
$$

Proof. By induction on $n$. The result is clearly true for $n=1$. Now assume it is true for all $n^{\prime}<n$; we prove it for $n^{\prime}=n$.

Write $n=2^{k}+j$, with $k \geq 1$ and $0 \leq j \leq 2^{k}-1$. We prove the identity by considering the high- and low-order terms of the polynomial $q_{j}^{X \mathcal{F}(e)}(X)$ separately. To do this, we consider the polynomial modulo $X^{2^{k}}$. First, we observe that

$$
\begin{aligned}
& q_{n}^{X \mathcal{F}(e)}(X)=K_{n}\left(a_{1}, \ldots, a_{n}\right) \\
& =K_{n}\left(a_{1}, \ldots, a_{2^{k}}, a_{2^{k}+1}, \ldots, a_{2^{k}+j}\right) \\
& =K_{n}\left(a_{1}, \ldots, a_{2^{k}},-a_{2^{k}-1}, \ldots,-a_{2^{k}-j}\right) \quad \text { (by Theorem 1(c)) } \\
& \equiv K_{n}\left(a_{1}, \ldots, a_{2^{k}-1}, 0,-a_{2^{k}-1}, \ldots,-a_{2^{k}-j}\right) \\
& \left(\bmod a_{2^{k}} K_{n-1}\left(a_{1}, \ldots, a_{2^{k}-1}\right)\right) \quad(\text { by Lemma } 2) \\
& \equiv K_{n}\left(a_{1}, \ldots, a_{2^{k}-1}, 0,-a_{2^{k}-1}, \ldots,-a_{2^{k}-j}\right)\left(\bmod X^{2^{k}}\right) \\
& \text { (by the induction hypothesis) } \\
& =K_{2^{k}-j-2}\left(a_{1}, \ldots, a_{2^{k}-j-2}\right)
\end{aligned}
$$

and so

$$
q_{n}^{X \mathcal{F}(e)}(X) \equiv q_{2^{k}-j-2}^{X \mathcal{F}(e)}(X)\left(\bmod X^{2^{k}}\right) .
$$

Now, recalling that $n=2^{k}+j$, define

$$
r_{j}(X)=\frac{q_{2^{k}+j}^{X \mathcal{F}(e)}(X)-q_{2^{k}-j-2}^{X \mathcal{F}(e)}(X)}{-e_{k} e_{k+1} X^{2^{k}}}
$$

for $0 \leq j \leq 2^{k}-1$. (We omit the superscript on the variable $r_{j}$, since it is the same in both of the terms in the numerator of the expression that defines it.)

By induction, we know that

$$
q_{2^{k}-1}^{X \mathcal{F}(e)}(X)=-e_{k} X^{2^{k}-1},
$$


so the sign of the leading coefficient of $q_{2^{k}}^{X \mathcal{F}(e)}$ is $-e_{k} e_{k+1}$. Hence $r_{0}(X)=1$. Similarly, it is easily verified that $r_{-1}(X)=0$. We claim that

$$
r_{j}(X)=a_{2^{k}+j} r_{j-1}(X)+r_{j-2}(X)
$$

for $1 \leq j \leq 2^{k}-1$. From the definition of $r_{j}(X)$, it suffices to verify that both $\overline{q_{2^{k}+j}^{X \mathcal{F}(e)}}(X)$ and $q_{2^{k}-j-2}^{X \mathcal{F}(e)}(X)$ satisfy this recurrence. The first is clear. For the second, observe that

$$
q_{2^{k}-j}=a_{2^{k}-j} q_{2^{k}-j-1}+q_{2^{k}-j-2},
$$

so

$$
q_{2^{k}-j-2}=-a_{2^{k}-j} q_{2^{k}-j-1}+q_{2^{k}-j} .
$$

But, by Theorem 1 (c), we have $a_{2^{k}-j}=-a_{2^{k}+j}$ for $1 \leq j \leq 2^{k}-1$. It follows that

as desired.

$$
q_{2^{k}-j-2}=a_{2^{k}+j} q_{2^{k}-j-1}+q_{2^{k}-j},
$$

Now, by the folding property,

$$
\begin{aligned}
\left(a_{2^{k}+1}, a_{2^{k}+2}, \ldots, a_{2^{k+1}-1}\right) & =\left(-a_{2^{k}-1},-a_{2^{k}-2}, \ldots,-a_{1}\right) \\
& =-X \mathcal{F}\left(e_{1}, \ldots, e_{k}\right)^{\mathrm{R}} \\
& =-X\left(\mathcal{F}\left(e_{1}, \ldots, e_{k-1}\right), e_{k},-\mathcal{F}\left(e_{1}, \ldots, e_{k-1}\right)^{\mathrm{R}}\right)^{\mathrm{R}} \\
& =X\left(\mathcal{F}\left(e_{1}, \ldots, e_{k-1}\right),-e_{k},-\mathcal{F}\left(e_{1}, \ldots, e_{k-1}\right)^{\mathrm{R}}\right) \\
& =X \mathcal{F}\left(e_{1}, \ldots, e_{k-1},-e_{k}\right),
\end{aligned}
$$

so we have $r_{j}(X)=q_{j}^{X \mathcal{F}\left(e_{1}, \ldots, e_{k-1},-e_{k}\right)}(X)$ for $0 \leq j \leq 2^{k}-1$.

Finally, it remains to see that $q_{2^{k+1}-1}^{X \mathcal{F}(e)}(X)=-e_{k+1} X^{2^{k+1}-1}$. To see this, note that we have shown that

$$
\begin{aligned}
q_{2^{k+1}-1}^{X \mathcal{F}(e)}(X) & =-e_{k} e_{k+1} X^{2^{k}} q_{2^{k}-1}^{X \mathcal{F}\left(e^{\prime}\right)}(X)+q_{-1}(X) \\
& =-e_{k} e_{k+1} X^{2^{k}} q_{2^{k}-1}^{X \mathcal{F}\left(e^{\prime}\right)}(X) \\
& =\left(-e_{k} e_{k+1} X^{2^{k}}\right)\left(e_{k} X^{2^{k}-1}\right)=-e_{k+1} X^{2^{k+1}-1},
\end{aligned}
$$

where we have used the fact that $q_{-1}(X)=0$, induction, and the fact that $e_{k}^{2}=1$. This completes the proof.

COROLlary 11. All the coefficients of $q_{n}^{X \mathcal{F}(e)}(X)$, no matter what choice of signs is made, are in $\{1,0,-1\}$.

Proof. An easy induction on $n$, using the fact that Theorem 10 writes $q_{n}(X)$ as the sum of a polynomial with lowest nonzero term of degree $X^{2^{k}}$ and one with highest nonzero term of degree $X^{2^{k}-1}$.

We now turn to a kind of converse to Corollary 11. It is easiest to phrase this converse in terms of something we call the infinite continuant tree $T$. 
The root of this infinite binary tree is labeled $K_{0}(0)=1$. If $z$ is a node of the tree labeled $K_{n-1}\left(a_{1}, \ldots, a_{n-1}\right)$, then there is an edge labeled +1 connecting $z$ to its left child, labeled $K_{n}\left(a_{1}, \ldots, a_{n-1}, X\right)$. There is also an edge labeled -1 connecting $z$ to its right child, labeled $K_{n}\left(a_{1}, \ldots, a_{n-1},-X\right)$. Figure 1 gives the first few levels of the tree $T$. Labels on the edges have been omitted.

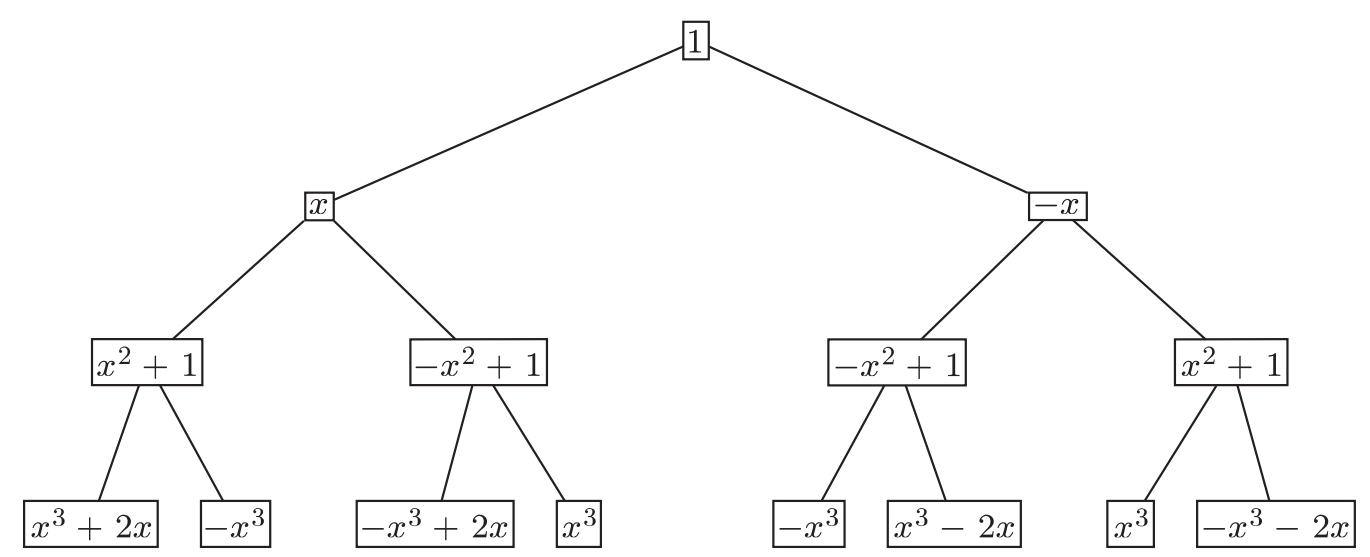

Fig. 1. The first 4 levels of the continuant tree

By Corollary 11, any infinite path labeled with a paperfolding sequence reaches nodes with labels that have only coefficients $0,1,-1$. The next theorem says that in $T$, only the paperfolding paths have this property.

TheOREm 12. Suppose we define a sequence of polynomials as follows: $q_{-1}(X)=0, q_{0}(X)=1$, and $q_{i}(X)=a_{i} X q_{i-1}(X)+q_{i-2}(X)$, where $a_{i} \in$ $\{1,-1\}$. Furthermore, choose $a_{i}$ successively so that the resulting polynomials $q_{i}(X)$ have coefficients in $\{-1,0,1\}$. Then $\left(a_{1}, a_{2}, \ldots\right)$ is a paperfolding sequence.

Pro of. Suppose, contrary to what we want to prove, that $a_{1}, a_{2}, \ldots$ is not a paperfolding sequence. Then there is some index, say $n$, such that $a_{1}, \ldots, a_{n}$ is not the prefix of any paperfolding sequence. Without loss of generality let $n$ be the smallest such, so that $a_{1}, \ldots, a_{n-1}$ is the prefix of some paperfolding sequence.

By assumption, $q_{n}=a_{n} X q_{n-1}+q_{n-2}$ has only coefficients $0,1,-1$, and by Theorem 10 so does $\widehat{q}_{n}=-a_{n} X q_{n-1}+q_{n-2}$. Write $n=2^{k}+j$, with $0 \leq$ $j<2^{k}$. If $n$ is not a power of 2 , then by reducing modulo $X^{2^{k}}$ and applying Theorem 10, we see that $q_{n-1}$ has a nonzero term of the form $\pm X^{2^{k}-j-1}$ and $q_{n-2}$ has a nonzero term of the form $\pm X^{2^{k}-j}$. Since $\widehat{q}_{n}=-a_{n} X q_{n-1}+q_{n-2}$, we see that these terms must cancel in $\widehat{q}_{n}$, or else we would have a coefficient of \pm 2 in the $X^{2^{k}-j}$ term in $\widehat{q}_{n}$. But then $X^{2^{k}-j}$ has a coefficient of \pm 2 in $q_{n}$, a contradiction. 
It follows that $n$ must be a power of 2 . But then either choice $a_{n}= \pm 1$ corresponds to a valid prefix of a paperfolding sequence, a contradiction.

In Figure 2 below, we display the first few levels of the infinite continuant tree with only the paperfolding paths shown.

The continuant tree $T$ has many other interesting properties, and we leave to the reader the pleasure of discovering some of them.

The reader will notice that in any particular level of Figure 2, the polynomials are essentially the same; only the signs of the terms differ. We now prove that this pattern continues throughout the continuant tree.

First, we define the support $\operatorname{supp}(b)$ of a polynomial $b(X)=b_{n} X^{n}+\ldots+$ $b_{1} X+b_{0}$ to be the set $\left\{i: b_{i} \neq 0\right\}$, that is, the set of exponents corresponding to nonzero coefficients. For example, $\operatorname{supp}\left(X^{5}-X\right)=\{1,5\}$. We also define $\left[X^{i}\right] b(X)$ to be $b_{i}$, the coefficient of the $X^{i}$ term in $b(X)$.

Corollary 13. Let $\mathcal{F}\left(e_{1}, e_{2}, \ldots\right)$ be a paperfolding sequence, and define $a=X \mathcal{F}\left(e_{1}, e_{2}, \ldots\right)$. Then the set $\operatorname{supp}\left(q_{n}^{a}(X)\right)$ is independent of $e_{1}, e_{2}, \ldots$, and is equal to

$$
\left\{n-2 i: 0 \leq i \leq n / 2,\left(\begin{array}{c}
n-i \\
i
\end{array}\right) \equiv 1(\bmod 2)\right\} .
$$

Proof. Since $e_{1}, e_{2}, \ldots$ is a paperfolding sequence, it follows that the coefficients of $q_{n}^{a}(X)$ are all $0,1,-1$. Now, taking everything mod 2, it follows that $q_{n}^{a}(X) \equiv s_{n}(X)(\bmod 2)$, where $s_{n}(X)$ is the denominator of the $n$th convergent to $[1, X, X, X, \ldots]$. But it is easy to prove by induction on $n$ that

$$
[X, X, X, \ldots]=\frac{t_{n+1}(X)}{t_{n}(X)}, \quad \text { where } t_{n}(X)=\sum_{0 \leq i \leq n / 2}\left(\begin{array}{c}
n-i \\
i
\end{array}\right) X^{n-2 i} .
$$

Clearly $s_{n}(X)=t_{n}(X)$, and the result follows.

Remark. If we define $a_{m, n}=\left[X^{n}\right] t_{m}(X) \bmod 2$, then it is not hard to see that

$$
a_{m, n}= \begin{cases}0 & \text { if } m \neq \equiv n(\bmod 2), \\
\left(\begin{array}{l}
m+n \\
m-n
\end{array}\right) \bmod 2 & \text { if } m \equiv n(\bmod 2) .\end{cases}
$$

Thus $\left(a_{m, n}\right)_{m, n \geq 0}$ is a 2-automatic double sequence in the sense of Salon $[17,18]$.

The size of the support of $q_{n}(X)$ can be computed using Theorem 10 . Define $u_{n}$ to be the number of nonzero coefficients in $q_{n}(X)$. Then we have

$$
u_{n}= \begin{cases}0 & \text { if } n=-1, \\ 1 & \text { if } n=0,1, \\ u_{j}+u_{2^{k}-j-2} & \text { if } n \geq 2 \text { and } n=2^{k}+j \text { with } 0 \leq j<2^{k} .\end{cases}
$$




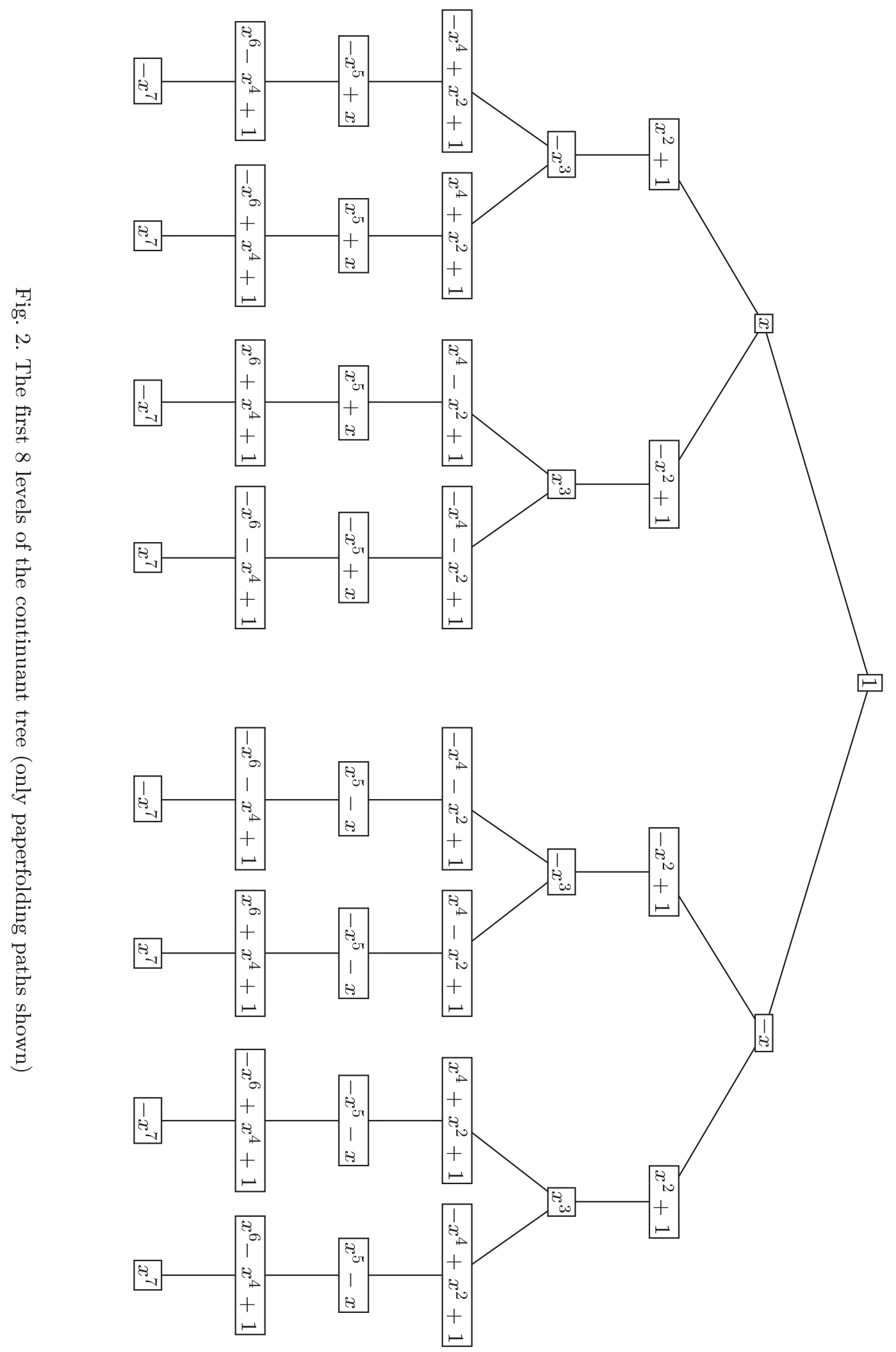


Here is a brief table of this sequence:

Table 3. The sequence $\left(u_{n}\right)$

\begin{tabular}{|c|r|r|r|r|r|r|r|r|r|r|r|r|r|r|r|r|r|r|}
\hline$n$ & -1 & 0 & 1 & 2 & 3 & 4 & 5 & 6 & 7 & 8 & 9 & 10 & 11 & 12 & 13 & 14 & 15 & 16 \\
\hline$u_{n}$ & 0 & 1 & 1 & 2 & 1 & 3 & 2 & 3 & 1 & 4 & 3 & 5 & 2 & 5 & 3 & 4 & 1 & 5 \\
\hline
\end{tabular}

This sequence is easily proved by induction to satisfy the identities

$$
u_{2 n}=u_{n}+u_{n-1} \quad(n \geq 1), \quad u_{2 n+1}=u_{n} \quad(n \geq 0) .
$$

The sequence $\left(u_{n}\right)_{n \geq 0}$ is the Stern-Brocot sequence [23,6], and is 2-regular in the sense of Allouche and Shallit [1]. It is Sloane and Plouffe's sequence M0141 [22].

6. Results through approximation. In this section we obtain more results on the convergents to $g_{\varepsilon}(X)$ and $h_{\varepsilon}(X)$ through some very simple approximation results. Our main tool is the the following theorem:

Theorem 14. Let $t(X)$ be a formal Laurent series with continued fraction expansion $\left[a_{0}, a_{1}, a_{2}, \ldots\right]$, let $p_{n} / q_{n}$ be the $n$th convergent, and let $p$ and $q$ be polynomials. Then

(a) $\operatorname{deg}\left(q_{n} t-p_{n}\right)=-\operatorname{deg} q_{n+1}<-\operatorname{deg} q_{n}$.

(b) If $\operatorname{deg}(q t-p)<-\operatorname{deg} q$, then $p / q$ is a convergent to $t$.

P r o o f. See, for example, [14]. Note that in our statement of part (b) of the theorem, we have omitted the superfluous condition, specified in [14], that the polynomials $p$ and $q$ be relatively prime.

Up to now, most of our results have been for $h_{\varepsilon}(X)$. We now show how to go from results for $h_{\varepsilon}(X)$ to results for $g_{\varepsilon}(X)$.

TheOREM 15. Let $p_{n} / q_{n}$ and $p_{n}^{\prime} / q_{n}^{\prime}$ be the $n$th convergents to $h_{\varepsilon}(X)=$ $X \sum_{i \geq 0} \varepsilon_{i} X^{-2^{i}}$ and $g_{\varepsilon}(X)=\sum_{i \geq 0} \varepsilon_{i} X^{-2^{i}}$ respectively. Then

$$
p_{n}^{\prime}=d_{n}\left(p_{n}-p_{n-1}\right) / X, \quad q_{n}^{\prime}=d_{n}\left(q_{n}-q_{n-1}\right),
$$

where $d_{n}= \pm 1$.

Proof. From Theorem 14(a), we obtain

$$
\begin{gathered}
\operatorname{deg}\left(q_{n} X g-p_{n}\right)=-\operatorname{deg} q_{n+1}=-(n+1) . \\
\operatorname{deg}\left(q_{n-1} X g-p_{n-1}\right)=-\operatorname{deg} q_{n}=-n .
\end{gathered}
$$

From (14) and (15), it follows that

$$
\operatorname{deg}\left(\left(q_{n}-q_{n-1}\right) X g-\left(p_{n}-p_{n-1}\right)\right)=-n,
$$

and so

$$
\operatorname{deg}\left(\left(q_{n}-q_{n-1}\right) g-\left(p_{n}-p_{n-1}\right) / X\right)=-(n+1) .
$$


Since $p_{i}(0)=1$ for all $i \geq-1$, it follows that $\left(p_{n}-p_{n-1}\right) / X$ is a polynomial. Since $\operatorname{deg} q_{n}^{\prime}=n$, it follows from Theorem 14(b) that

$$
\frac{\left(p_{n}-p_{n-1}\right) / X}{q_{n}-q_{n-1}}
$$

is the $n$th convergent to $g$. Since the degrees of numerator and denominator agree with the degrees of $p_{n}^{\prime}$ and $q_{n}^{\prime}$, it follows that

$$
p_{n}^{\prime}=d_{n}\left(p_{n}-p_{n-1}\right) / X, \quad q_{n}^{\prime}=d_{n}\left(q_{n}-q_{n-1}\right)
$$

for some sequence $\left(d_{n}\right)$ of \pm 1 's.

Remark. It is easy to determine precisely what the sequence $\left(d_{n}\right)$ is. Since the sign of the leading term of $p_{n}^{\prime}$ is clearly +1 , and the sign of the leading term of $p_{n}$ is $\prod_{1 \leq j \leq n} b_{j}$, where $\left(b_{1}, b_{2}, \ldots\right)=\mathcal{F}\left(\varepsilon_{1},-\varepsilon_{2},-\varepsilon_{3}, \ldots\right)$, it follows that $d_{n}=\prod_{1 \leq j \leq n} b_{j}$.

Corollary 16. The coefficients of $q_{n}^{\prime}(X)$ lie in $\{-1,0,1\}$ for all $n \geq 0$.

Proof. We know that $q_{n}^{\prime}= \pm\left(q_{n}-q_{n-1}\right)$, and it is easy to see $q_{2 n}(X)$ is an even polynomial and $q_{2 n+1}(X)$ is an odd polynomial. The result follows.

7. Further approximation results. Let $\mathcal{E}=\left\{\left(\varepsilon_{n}\right)_{n \geq 0} \in\{-1,+1\}^{\mathbb{N}}\right.$ : $\left.\varepsilon_{0}=1\right\}$. Define on $\mathcal{E}$ the map $T$ as follows:

$$
T\left(\left(\varepsilon_{n}\right)_{n \geq 0}\right)=\left(\varepsilon_{1} \varepsilon_{n}\right)_{n \geq 1}=\left(\varepsilon_{1} \varepsilon_{n+1}\right)_{n \geq 0} .
$$

For $\left(\varepsilon_{n}\right)_{n \geq 0} \in \mathcal{E}$, let $h_{\varepsilon}=X \sum_{k \geq 0} \varepsilon_{k} X^{-2^{k}}$. We know that the continued fraction expansion of $h_{\varepsilon}$ is of the form $[1, \pm X, \pm X, \ldots]$. Denote by $p_{\varepsilon, n}(X) / q_{\varepsilon, n}(X)$ its $n$th convergent. (Note that this notation is slightly different from the notation we introduced in Section 5. In particular, here the $\varepsilon$ indicates the dependence of the convergent on a particular choice of signs in the associated series, while in Section 5, the superscript indicated the dependence of the convergent on the particular choice of partial quotients.)

LEMma 17. The following properties hold:

(a) $\operatorname{deg} q_{\varepsilon, n}=n$,

(b) $\operatorname{deg}\left(h_{\varepsilon}(X)-p_{\varepsilon, n} / q_{\varepsilon, n}\right)=-2 n-1$.

Proof. As the continued fraction expansion of $h_{\varepsilon}$ is of the form $[1, \pm X$, $\pm X, \ldots]$, we have

$$
\begin{aligned}
p_{\varepsilon, n+2}(X) & = \pm X p_{\varepsilon, n+1}(X)+p_{\varepsilon, n}(X), \\
q_{\varepsilon, n+2}(X) & = \pm X q_{\varepsilon, n+1}(X)+q_{\varepsilon, n}(X),
\end{aligned}
$$

which proves our claim.

THEOREM 18. The following relations hold between the polynomials $q_{\varepsilon, 2 n}(X), q_{\varepsilon, 2 n+1}(X)$ and the polynomial $q_{T \varepsilon, n}(X)$ : 
(a) $q_{\varepsilon, 2 n+1}(X)=\varepsilon_{1} X q_{T \varepsilon, n}\left(X^{2}\right)$,

(b) $q_{\varepsilon, 2 n}(X)=(-1)^{n}\left(q_{T \varepsilon, n}\left(X^{2}\right)-q_{T \varepsilon, n-1}\left(X^{2}\right)\right)$,

(c) the polynomial $q_{\varepsilon, 2 n+1}(X)$ is odd,

(d) the polynomial $q_{\varepsilon, 2 n}(X)$ is even.

Proof. We have

$$
\begin{aligned}
\operatorname{deg}\left(h_{T \varepsilon}\left(X^{2}\right)-\frac{p_{T \varepsilon, n}\left(X^{2}\right)}{q_{T \varepsilon, n}\left(X^{2}\right)}\right) & =2 \operatorname{deg}\left(h_{T \varepsilon}(X)-\frac{p_{T \varepsilon, n}(X)}{q_{T \varepsilon, n}(X)}\right) \\
& =-4 n-2 .
\end{aligned}
$$

On the other hand, we have

$$
h_{T \varepsilon}\left(X^{2}\right)=X^{2} \sum_{k \geq 0} \varepsilon_{1} \varepsilon_{k+1} X^{-2^{k+1}}=\varepsilon_{1} X\left(h_{\varepsilon}(X)-1\right) .
$$

Combining (17) and (18), we get

$$
\operatorname{deg}\left(h_{\varepsilon}(X)-1-\varepsilon_{1} \frac{p_{T \varepsilon, n}\left(X^{2}\right)}{X q_{T \varepsilon, n}\left(X^{2}\right)}\right)=-4 n-3 .
$$

Now, considering the even- and odd-indexed convergents of $h_{\varepsilon}$, we get

$$
\begin{aligned}
\operatorname{deg}\left(h_{\varepsilon}(X)-\frac{p_{\varepsilon, 2 n}(X)}{q_{\varepsilon, 2 n}(X)}\right) & =-4 n-1, \\
\operatorname{deg}\left(h_{\varepsilon}(X)-\frac{p_{\varepsilon, 2 n+1}(X)}{q_{\varepsilon, 2 n+1}(X)}\right) & =-4 n-3 .
\end{aligned}
$$

Now, combining (19) and (20), we get

Hence

$$
\begin{aligned}
\operatorname{deg}\left(\frac{p_{\varepsilon, 2 n}(X)}{q_{\varepsilon, 2 n}(X)}-1-\varepsilon_{1} \frac{p_{T \varepsilon, n}\left(X^{2}\right)}{X q_{T \varepsilon, n}\left(X^{2}\right)}\right) & =-4 n-1, \\
\operatorname{deg}\left(\frac{p_{\varepsilon, 2 n+1}(X)}{q_{\varepsilon, 2 n+1}(X)}-1-\varepsilon_{1} \frac{p_{T \varepsilon, n}\left(X^{2}\right)}{X q_{T \varepsilon, n}\left(X^{2}\right)}\right) & \leq-4 n-3 .
\end{aligned}
$$

$$
\begin{gathered}
\operatorname{deg}\left(X q_{T \varepsilon, n}\left(X^{2}\right) p_{\varepsilon, 2 n}(X)-X q_{\varepsilon, 2 n}(X) q_{T \varepsilon, n}\left(X^{2}\right)-\varepsilon_{1} p_{T \varepsilon, n}\left(X^{2}\right) q_{\varepsilon, 2 n}(X)\right)=0 \\
\operatorname{deg}\left(X q_{T \varepsilon, n}\left(X^{2}\right) p_{\varepsilon, 2 n+1}(X)-X q_{\varepsilon, 2 n+1}(X) q_{T \varepsilon, n}\left(X^{2}\right)\right. \\
\left.-\varepsilon_{1} p_{T \varepsilon, n}\left(X^{2}\right) q_{\varepsilon, 2 n+1}(X)\right) \leq-1
\end{gathered}
$$

We thus have two polynomials, one of which has degree 0 , hence is constant and equal to its value at 0 , the second of which has degree at most -1 , hence $-\infty$ and hence is the zero polynomial. Using the values at 0 of the $q$ 's and $p$ 's that we gave in Theorem 5 , we get

$$
\begin{gathered}
X q_{T \varepsilon, n}\left(X^{2}\right) p_{\varepsilon, 2 n}(X)-X q_{\varepsilon, 2 n}(X) q_{T \varepsilon, n}\left(X^{2}\right)-\varepsilon_{1} p_{T \varepsilon, n}\left(X^{2}\right) q_{\varepsilon, 2 n}(X)=-\varepsilon_{1}, \\
X q_{T \varepsilon, n}\left(X^{2}\right) p_{\varepsilon, 2 n+1}(X)-X q_{\varepsilon, 2 n+1}(X) q_{T \varepsilon, n}\left(X^{2}\right) \\
-\varepsilon_{1} p_{T \varepsilon, n}\left(X^{2}\right) q_{\varepsilon, 2 n+1}(X)=0 .
\end{gathered}
$$


Solving this linear system in $q_{T \varepsilon, n}\left(X^{2}\right)$ and $p_{T \varepsilon, n}\left(X^{2}\right)$ and noticing that, by (3), its determinant

$$
\varepsilon_{1} X p_{\varepsilon, 2 n+1}(X) q_{\varepsilon, 2 n}(X)-p_{\varepsilon, 2 n}(X) q_{\varepsilon, 2 n+1}(X)
$$

is equal to $\varepsilon_{1} X$, we obtain

$$
\begin{aligned}
& q_{\varepsilon, 2 n+1}(X)=\varepsilon_{1} X q_{T \varepsilon, n}\left(X^{2}\right), \\
& p_{\varepsilon, 2 n+1}(X)=p_{T \varepsilon, n}\left(X^{2}\right)+\varepsilon_{1} X q_{T \varepsilon, n}\left(X^{2}\right) .
\end{aligned}
$$

This proves parts (a) and (c) of our theorem.

Now, using the recurrence relation satisfied by the $q_{n}$ 's and the fact that the partial quotients of $h_{\varepsilon}$ are $\pm X$, we get

$$
q_{\varepsilon, 2 n+1}(X)= \pm X q_{\varepsilon, 2 n}(X)+q_{\varepsilon, 2 n-1}(X) .
$$

Replacing $q_{\varepsilon, 2 n+1}(X)$ (and $\left.q_{\varepsilon, 2 n-1}(X)\right)$ by the expressions in $q_{T \varepsilon, n}\left(X^{2}\right)$ (and $\left.q_{T \varepsilon, n-1}\left(X^{2}\right)\right)$ we have just obtained gives

$$
\varepsilon_{1} X q_{T \varepsilon, n}\left(X^{2}\right)= \pm X q_{\varepsilon, 2 n}(X)+\varepsilon_{1} X q_{T \varepsilon, n-1}\left(X^{2}\right),
$$

that is,

$$
q_{\varepsilon, 2 n}(X)= \pm \varepsilon_{1}\left(q_{T \varepsilon, n}\left(X^{2}\right)-q_{T \varepsilon, n-1}\left(X^{2}\right)\right) .
$$

Looking at the value at 0 , we get

$$
q_{\varepsilon, 2 n}(X)=(-1)^{n}\left(q_{T \varepsilon, n}\left(X^{2}\right)-q_{T \varepsilon, n-1}\left(X^{2}\right)\right) .
$$

This proves parts (b) and (d) of our theorem.

R e mark. Theorem 18 can be used to give a different proof of Corollary 11. Indeed, we deduce from Theorem 18 that if the coefficients of $q_{\varepsilon, j}$ are all $0, \pm 1$ for all $j \leq n$, then the coefficients of $q_{\varepsilon, 2 n}$ and $q_{\varepsilon, 2 n+1}$ have the same property: the different parities of $q_{\varepsilon, n}$ and $q_{\varepsilon, n-1}$ ensure that there is no "overlap" in the equation of Theorem 18(b).

We conclude the paper by proving a results about the automaticity of the double sequence $\left(\left[X^{n}\right] q_{m, \varepsilon}(X)\right)_{m, n \geq 0}$.

TheOREM 19. Let $\left(\varepsilon_{n}\right)_{n \geq 0}$ be a sequence in $\mathcal{E}$. As before, let $p_{\varepsilon, n}(X) / q_{\varepsilon, n}(X)$ be the nth convergent of the continued fraction expansion of $h_{\varepsilon}=X \sum_{k \geq 0} \varepsilon_{k} X^{-2^{k}}$. Let $\left(a_{m, n}^{\varepsilon}\right)_{m, n \geq 0}$ be the double sequence defined by $a_{m, n}=\left[X^{n}\right] q_{m, \varepsilon}(X)$. Then the double sequence $\left(a_{m, n}^{\varepsilon}\right)_{m, n \geq 0}$ is 2-automatic in the sense of Salon $[17,18]$ if and only if the sequence $\varepsilon=\left(\varepsilon_{j}\right)_{j \geq 0}$ is ultimately periodic.

Proof. Suppose first that the sequence $\left(a_{m, n}^{\varepsilon}\right)_{m, n \geq 0}$ is 2-automatic. Then $[17,18]$ the sequence $\left(a_{n, n}^{\varepsilon}\right)_{n \geq 0}$ is a one-dimensional 2-automatic sequence. Hence, by a classical result, the sequence

is ultimately periodic.

$$
\left(a_{2^{j}-1,2^{j}-1}^{\varepsilon}\right)_{j \geq 0}
$$


But from Theorem 18 we have

$$
a_{2 m+1,2 n+1}^{\varepsilon}=\varepsilon_{1} a_{m, n}^{T \varepsilon} .
$$

By induction, it follows that

$$
a_{2^{j}-1,2^{j}-1}^{\varepsilon}=\varepsilon_{1}(T \varepsilon)_{1} \ldots\left(T^{j-1} \varepsilon\right)_{1} a_{0,0}^{T^{j} \varepsilon} .
$$

Now observe that $a_{0,0}^{T^{j} \varepsilon}=1$ and $\left(T^{j} \varepsilon\right)_{n}=\varepsilon_{j} \varepsilon_{n+j}$. Hence

$$
\varepsilon_{1}(T \varepsilon)_{1} \ldots\left(T^{j-1} \varepsilon\right)_{1}=\varepsilon_{j}=a_{2^{j}-1,2^{j}-1}^{\varepsilon} .
$$

Since $\left(a_{2^{j}-1,2^{j}-1}^{\varepsilon}\right)_{j \geq 0}$ is ultimately periodic, the sequence $\left(\varepsilon_{j}\right)_{j \geq 0}$ is also ultimately periodic.

Now we prove the converse. Suppose that the sequence $\left(\varepsilon_{j}\right)_{j \geq 0}$ is ultimately periodic. Theorem 18 gives the following relations:

$$
\begin{aligned}
a_{2 m, 2 n}^{\varepsilon} & =(-1)^{m}\left(a_{m, n}^{T \varepsilon}-a_{m-1, n}^{T \varepsilon}\right), & a_{2 m+1,2 n}^{\varepsilon} & =0, \\
a_{2 m, 2 n+1}^{\varepsilon} & =0, & a_{2 m+1,2 n+1}^{\varepsilon} & =\varepsilon_{1} a_{m, n}^{T \varepsilon} .
\end{aligned}
$$

Denote by $V_{m, n}^{\varepsilon}$ for $m \geq 1$ the vector

We then obtain

$$
V_{m, n}^{\varepsilon}=\left(\begin{array}{c}
a_{m, n}^{\varepsilon} \\
a_{m-1, n}^{\varepsilon} \\
(-1)^{m} a_{m, n}^{\varepsilon} \\
(-1)^{m} a_{m-1, n}^{\varepsilon}
\end{array}\right)
$$

$$
\begin{aligned}
V_{2 m, 2 n}^{\varepsilon} & =A_{0,0} V_{m, n}^{T \varepsilon}, & V_{2 m+1,2 n}^{\varepsilon} & =A_{1,0} V_{m, n}^{T \varepsilon} \\
V_{2 m, 2 n+1}^{\varepsilon} & =\varepsilon_{1} A_{0,1} V_{m, n}^{T \varepsilon}, & V_{2 m+1,2 n+1}^{\varepsilon} & =\varepsilon_{1} A_{1,1} V_{m, n}^{T \varepsilon}
\end{aligned}
$$

where the matrices $A_{i, j}$ are given by

$$
\begin{aligned}
A_{0,0} & =\left(\begin{array}{cccc}
0 & 0 & 1 & -1 \\
0 & 0 & 0 & 0 \\
0 & 0 & 1 & -1 \\
0 & 0 & 0 & 0
\end{array}\right), & A_{0,1} & =\left(\begin{array}{cccc}
0 & 0 & 0 & 0 \\
0 & 1 & 0 & 0 \\
0 & 0 & 0 & 0 \\
0 & 1 & 0 & 0
\end{array}\right), \\
A_{1,0} & =\left(\begin{array}{cccc}
0 & 0 & 0 & 0 \\
0 & 0 & 1 & -1 \\
0 & 0 & 0 & 0 \\
0 & 0 & -1 & 1
\end{array}\right), & A_{1,1} & =\left(\begin{array}{cccc}
1 & 0 & 0 & 0 \\
0 & 0 & 0 & 0 \\
-1 & 0 & 0 & 0 \\
0 & 0 & 0 & 0
\end{array}\right) .
\end{aligned}
$$

Now, examining the set of subsequences of the double sequence $\left(V_{m, n}^{\varepsilon}\right)_{m, n}$ defined by

$$
\mathcal{N}=\left\{\left(V_{2^{j} m+r, 2^{j} n+s}^{T^{u} \varepsilon}\right)_{m, n \geq 0}: j \geq 0,0 \leq r, s \leq 2^{j}-1, u \geq 0\right\}
$$

we see from the above relations that this set is finite if $\varepsilon=T^{j} \varepsilon$ for some $j$, i.e., if the sequence $\varepsilon$ is ultimately periodic. Hence the 2-kernel of the 
sequence $\left(V_{m, n}^{\varepsilon}\right)_{m, n}$ is also finite, which means that this sequence is 2automatic. Finally, its first component $\left(a_{m, n}^{\varepsilon}\right)_{m, n}$ is also 2-automatic.

R e mark. The same proof works for the coefficients of the polynomial $q_{\varepsilon, n}^{\prime}$, but the corresponding result for the coefficients of $q_{\varepsilon, n}^{\prime}$ can also be deduced from Theorem 15 and from the property that $\left(d_{n}\right)_{n}$ is 2-automatic if and only if $\left(\varepsilon_{n}\right)_{n}$ is ultimately periodic.

Acknowledgments. The first and third authors wish to thank the Fields Institute for its hospitality during the workshop on Aperiodic Long Range Order held in Waterloo in August-September 1995. Part of the work of the third author was done under the auspices of the Mahler lectureship in Australia; that and the work of the fourth author was partly supported by grants from the Australian Research Council.

\section{References}

[1] J.-P. Allouche and J. O. Shallit, The ring of k-regular sequences, Theoret. Comput. Sci. 98 (1992), 163-187.

[2] E. Artin, Quadratische Körper im Gebiet der höheren Kongruenzen I, II, Math. Z. 19 (1924), 153-246. Reprinted in Collected Papers, pp. 1-104.

[3] L. E. Baum and M. M. Sweet, Continued fractions of algebraic power series in characteristic 2, Ann. of Math. 103 (1976), 593-610.

[4] A. Blanchard and M. Mendès France, Symétrie et transcendance, Bull. Sci. Math. 106 (1982), 325-335.

[5] F. M. Dekking, M. Mendès France and A. J. van der Poorten, Folds!, Math. Intelligencer 4 (1982), 130-138, 173-181, 190-195.

[6] R. L. Graham, D. E. Knuth and O. Patashnik, Concrete Mathematics, AddisonWesley, 1989.

[7] G. H. Hardy and E. M. Wright, An Introduction to the Theory of Numbers, 5th ed., Oxford University Press, 1989.

[8] M. Kmošek, Continued fraction expansion of some irrational numbers, Master's thesis, Uniwersytet Warszawski, 1979 (in Polish).

[9] D. E. Knuth, Seminumerical Algorithms, Vol. II of The Art of Computer Programming, Addison-Wesley, 1981.

[10] G. Köhler, Some more predictable continued fractions, Monatsh. Math. 89 (1980), 95-100.

[11] B. de Mathan, Approximations diophantiennes dans un corps local, Bull. Soc. Math. France Suppl. Mém. 21 (1970), 1-93.

[12] M. Mendès France, Principe de la symétrie perturbée, in: M.-J. Bertin (ed.), Séminaire de Théorie des Nombres, Paris 1979-80; Séminaire Delange-Pisot-Poitou, Birkhäuser, 1981, 77-98.

[13] M. Mendès France and A. J. van der Poorten, Arithmetic and analytic properties of paper folding sequences, Bull. Austral. Math. Soc. 24 (1981), 123-131.

[14] - - - Some explicit continued fraction expansions, Mathematika 38 (1991), 1-9.

[15] A. J. van der Poorten and J. O. Shallit, Folded continued fractions, J. Number Theory 40 (1992), 237-250. 
[16] J. Roberts, Elementary Number Theory: A Problem Oriented Approach, MIT Press, 1978.

[17] O. Salon, Suites automatiques à multi-indices et algébricité, C. R. Acad. Sci. Paris 305 (1987), 501-504.

[18] —, Propriétés arithmétiques des automates multidimensionnels, $\mathrm{PhD}$ thesis, Université de Bordeaux I, 1989.

[19] J. O. Shallit, Simple continued fractions for some irrational numbers, J. Number Theory 11 (1979), 209-217.

[20] - Explicit descriptions of some continued fractions, Fibonacci Quart. 20 (1982), $77-81$.

[21] —, Simple continued fractions for some irrational numbers, II, J. Number Theory 14 (1982), 228-231.

[22] N. J. A. Sloane and S. Plouffe, The Encyclopedia of Integer Sequences, Academic Press, 1995.

[23] M. A. Stern, Über eine zahlentheoretische Funktion, J. Reine Angew. Math. 55 (1858), 193-220.

\section{CNRS}

LRI

Bâtiment 490

F-91405 Orsay Cedex, France

E-mail: allouche@lri.lri.fr

Mathématiques et Informatique

Université Bordeaux I

351 , cours de la Libération

F-33405 Talence Cedex, France

E-mail:mmf@math.u-bordeaux.fr

Department of Computer Science

University of Waterloo

Waterloo, Ontario N2L 3G1

Canada

E-mail: shallit@graceland.uwaterloo.ca
Department of Computer Science

University of Waterloo Waterloo, Ontario N2L 3G1

Canada

E-mail: alubiw@waterloo.edu

School of MPCE

Macquarie University

North Ryde, New South Wales 2109

Australia

E-mail: alf@mpce.mq.edu.au 